\title{
Mechanism behind Hyperkalemic Brugada Phenocopy: A Computational Study
}

\author{
Ismael Hernández-Romero ${ }^{1,2}$, Paula Giménez ${ }^{2}$, Allan Rivera², Carlos Figura ${ }^{1}$, Maria S Guillem³ \\ Francisco Fernández-Avilés ${ }^{2}$, Andreu M Climent ${ }^{2}$, Felipe Atienza ${ }^{2}$ \\ ${ }^{1}$ Department of Telecommunication Engineering, Universidad Rey Juan Carlos, Fuenlabrada, Spain \\ ${ }^{2}$ Cardiology Department, Hospital General Universitario Gregorio Marañón, Instituto de \\ Investigación Sanitaria Gregorio Marañón, Madrid, Spain \\ ${ }^{3}$ ITACA, Universitat Politècnica de Valencia, Valencia, Spain
}

\begin{abstract}
Brugada Phenocopy (BrP) describes an ECG pattern that imitates the morphology of a congenital Brugada Syndrome, but is caused by various other factors, such as metabolic abnormalities, ischemia or others. Understanding the underlying mechanisms of $\mathrm{BrP}$ can improve the outcome of these patients. This study proposes an explanation to $\mathrm{BrP}$ cases elicited by hyperkalemia. A $3 D$ wedge model of right ventricle was implemented, along with a transmural cell gradient of endocardial, mid-myocardial and epicardial cell. Two protocols of stimulation were designed to introduce a delay of activation in the model. Computer simulations were performed increasing the amount of extracellular potassium from healthy stage to hyperkalemic condition and pseudoECGs were calculated.

Delay of activation and heterogeneity of conduction velocity are linked of Brugada Phenocopy in a hyperkalemic stage. Increasing extracellular potassium promotes these differences, due to elevation of resting potential and lower availability of sodium channels.
\end{abstract}

\section{Introduction}

Brugada syndrome is commonly defined as a hereditary cardiac disorder characterized by a ST-segment elevation in the right precordial leads of the electrocardiogram (ECG), and a propensity for sudden cardiac death [1]. However, it has been well recognized that other factors can promote a manifestation of Brugada syndrome in patients who do not have the genetically determined disease [1]. The acquired Brugada-like ECG pattern is denominated Brugada phenocopy $(\mathrm{BrP})[2,3]$. Currently, a classification of 6 categories of BrP exists: metabolic conditions, mechanical compression, ischemia and pulmonary embolism, myocardial and pericardial disease, ECG modulation and miscellaneous [3].

Hyperkalemia, an elevated level of the serum potassium in the blood, belongs at group of metabolic conditions. In clinical practice, hyperkalemia produces changes in ECG as disappearing of P wave, narrowing of QT, high narrow $\mathrm{T}$ waves, atrioventricular blocks and bundle branch blocks [4]. However, several cases have been reported as $\mathrm{BrP}$ in patients with hyperkalemia [5].

Actually, several theories seek to explain the mechanism that produce Brugada pattern, either product of a congenital disease or different factors of a BrP. The main two are the depolarization theory and the repolarization theory [6].

The depolarization theory supports the idea that the ST-segment elevation is caused by the conduction delay in the right ventricular outflow tract (RVOT) [6,7]. The delayed depolarization of the RVOT with respect to the rest of the right ventricle creates a potential difference more negative on the onset of the depolarization and more positive at the end of action potential. On the other hand, the repolarization theory hypothesizes that reduced inward sodium current and increased transient outward potassium current generate a larger notch in the action potential in the right ventricular epicardium, which produces a transmural voltage gradient and the STsegment elevation [6,7].

In this work, we have studied if the depolarization theory is the reason behind the $\mathrm{BrP}$ in patients with hyperkalemia using a computational wedge model.

\section{Methods}

The electrical activity of the cardiac tissue was modeled using the following differential equations:

$$
\begin{gathered}
\frac{\partial V}{\partial t}=\nabla \cdot D V V-\frac{\left(I_{\text {ion }}+I_{\text {stim }}\right)}{C_{\mathrm{m}}} \\
\frac{\partial u}{\partial t}=f(u, V, t)
\end{gathered}
$$


where $V$ is the transmembrane potential, $\mathrm{D}$ is the diffusion coefficient, $C_{m}$ the membrane capacitance, Iion the ionic current, $I$ stm the stimulus current, and $\mathrm{u}$ is a set of state variables associated with the ionic model. Anisotropic conduction was generated using the original fiber directions of the geometrical model.

Three cell types were modelled using a modified version of the O'Hara-Rudy dynamic (ORd) human model [8]; Endocardial cells (Endo), M-cells (M) and Epicardial cells (Epi), in which the maximum conductances of some currents were adjust to obtain a realistic ECG pattern. $\mathrm{G}_{\mathrm{Kr}}$ was scaled to $35 \%$ of original [8]. Scaling factors of cell types are shown in tab. 1.

Table 1. Scaling Factors for Model Implementation of Transmural Heterogeneity.

\begin{tabular}{ccc}
\hline Parameter & Epi/Endo & M/Endo \\
\hline $\mathrm{G}_{\mathrm{Nal}}$ & 0.6 & 1 \\
$\mathrm{G}_{\mathrm{to}}$ & 4.0 & 4.0 \\
$\mathrm{P}_{\mathrm{Ca}}$ & 1.2 & 2.5 \\
$\mathrm{G}_{\mathrm{Kr}}$ & 9.1 & 0.8 \\
$\mathrm{G}_{\mathrm{Ks}}$ & 1.4 & 1 \\
$\mathrm{G}_{\mathrm{K} 1}$ & 1.2 & 1.3 \\
$\mathrm{G}_{\mathrm{NaCa}}$ & 1.1 & 1.4 \\
$\mathrm{G}_{\mathrm{NaK}}$ & 0.9 & 0.7 \\
$\mathrm{G}_{\mathrm{Kb}}$ & 0.6 & 1 \\
\hline
\end{tabular}

A geometrical wedge model was created from the anatomic model described in [9]. The dimensions of the wedge model were resized to $24 \times 30 \times 5 \mathrm{~mm}$, and it was composed of approximately 50,000 cells. A transmural gradient of the three cell types was incorporated adding an endocardical, M-cell and epicardical layer equally distributed across the total transmural length. Figure 1 shows the transmural distribution of the cardiac wedge model.
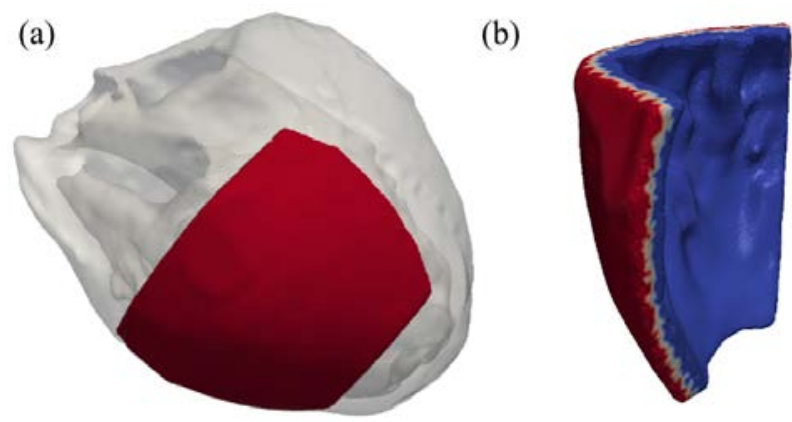

Figure 1. Geometrical model a) Wedge created from original model, b) Cell distribution of the transmural gradient: Endo (blue), M-cells (white) and Epi (red).

Two protocols of stimulation were designed to evaluate the effect of depolarization theory and conduction delay. In the protocol 1, all the cells of the layer more endocardical were stimulated; and in the protocol 2, only $60 \%$ of the bottom part of cells chosen at protocol 1 was paced. The configurations of the protocols are shown in Figure 2.

Simulations with both stimulation protocols were performed with three different concentrations of extracellular potassium $\left(\left[\mathrm{K}^{+}\right]_{\mathrm{o}}\right)$ : basal conditions with $\left[\mathrm{K}^{+}\right]_{\mathrm{o}}=5.4 \mathrm{mM}$, intermediate stage with $\left[\mathrm{K}^{+}\right]_{\mathrm{o}}=7 \mathrm{mM}$ and hyperkalemic stage with $\left[\mathrm{K}^{+}\right]_{\mathrm{o}}=10.0 \mathrm{mM}$. (a)

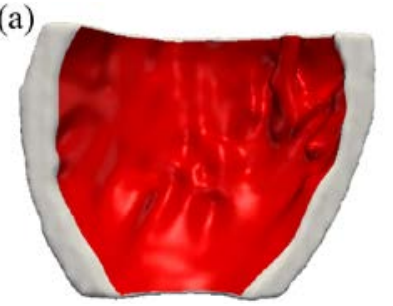

(b)

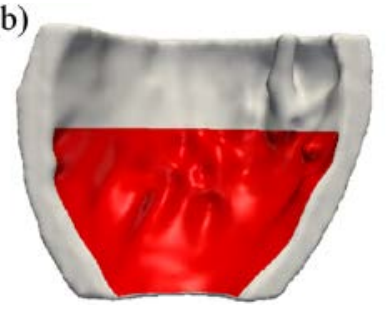

Figure 2. Protocols of stimulation a) Whole endocardical layer, b) $60 \%$ of endocardical layer.

Computations were performed on a GPU Tesla K20 (5GRAM DDR5) [10] using Forward-Euler algorithm with a fixed time step of $0.01 \mathrm{~ms}$.

Pseudo-electrograms (EGM) were computed by using transmembrane potentials according to Equation (3) using the position that V1 occupies of the standard 12 lead ECG and $3 \mathrm{~cm}$ distance from the epicardial surface [11]:

$$
E G M=\sum_{\vec{y}}\left(\frac{\vec{r}}{r^{3}}\right) \cdot \vec{\nabla} V_{m}
$$

where $\vec{r}$ is the distance vector between the measuring point and a point in the tissue domain, $\vec{\nabla}$ denotes the gradient operator, and $\mathrm{Vm}$ is the transmembrane potential.

Changes in morphology of waves of pseudo-ECG, isochrones maps over the model and spatial conduction velocity $(\mathrm{CV})$ were quantified.

\section{Results}

The gradually increment of $\left[\mathrm{K}^{+}\right]_{\mathrm{o}}$ in the simulations had shown changes in activation patterns. Effects of reduction of $\mathrm{CV}$, during the application of endocardical pacing, were larger when less endocardical area was stimulated in protocol 2. Simulations with this protocol achieved a CV of $75 \mathrm{~cm} / \mathrm{s}$ in basal conditions with $\left[\mathrm{K}^{+}\right]_{\mathrm{o}}=$ $5.4 \mathrm{mM}, 24 \mathrm{~cm} / \mathrm{s}$ with $\left[\mathrm{K}^{+}\right]_{\mathrm{o}}=7 \mathrm{mM}$ and $20 \mathrm{~cm} / \mathrm{s}$ with $\left[\mathrm{K}^{+}\right]_{\mathrm{o}}=10.0 \mathrm{mM}$. The activation patterns are shown in Figure 3.

In the action potentials, the effect of an elevated $\left[\mathrm{K}^{+}\right]_{\mathrm{o}}$ concentration increased the resting potential, from -87.67 
(a)

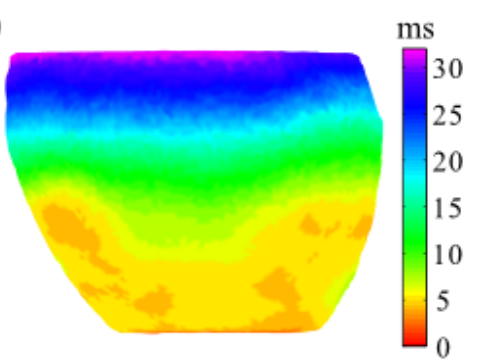

(b)

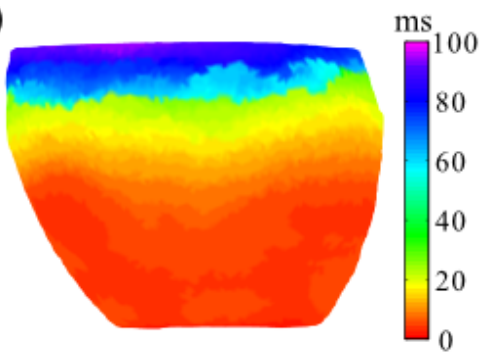

(c)

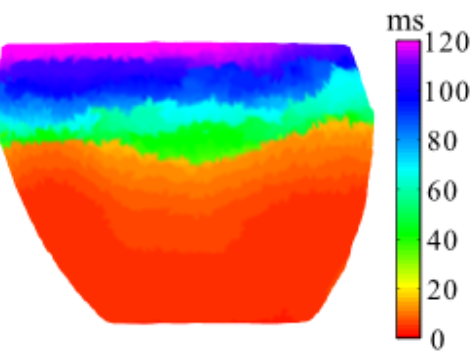

Figure 3. Activation patterns of simulated data with protocol 2 a) Basal conditions with $\left[\mathrm{K}^{+}\right]_{\mathrm{o}}=5.4 \mathrm{mM}$, b) Intermediate with $\left.\left[\mathrm{K}^{+}\right]_{\mathrm{o}}=7 \mathrm{mM}, \mathrm{c}\right)$ Hyperkalemic stage with $\left[\mathrm{K}^{+}\right]_{\mathrm{o}}=10.0 \mathrm{mM}$.

(a)

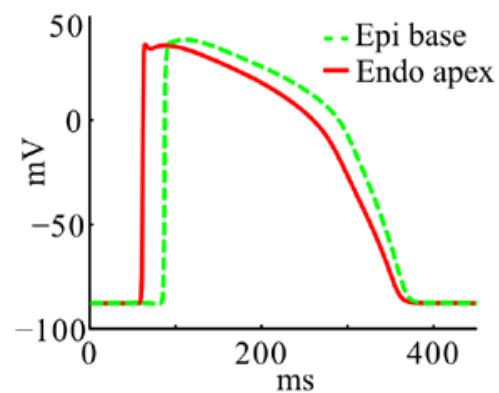

(b)

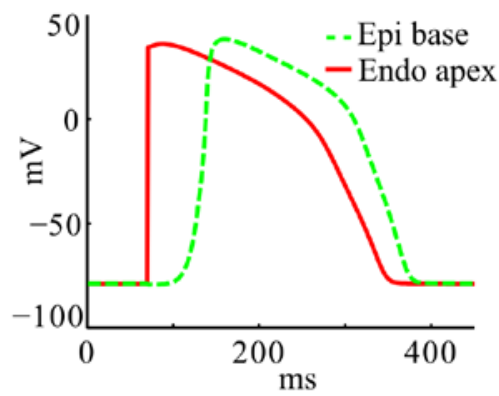

(c)

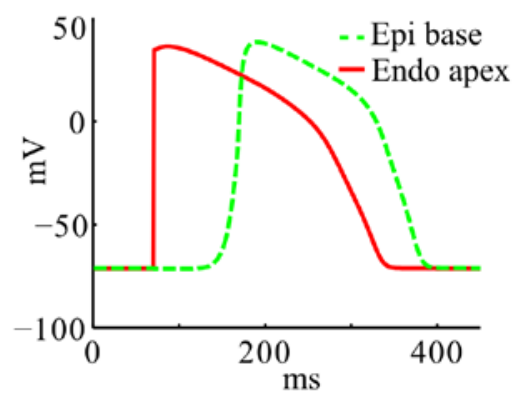

Figure 4. Action potentials from epicardical upper part (green) and endocardical bottom part of model (red) a) Basal conditions with $\left[\mathrm{K}^{+}\right]_{\mathrm{o}}=5.4 \mathrm{mM}$, b) Intermediate with $\left[\mathrm{K}^{+}\right]_{\mathrm{o}}=7 \mathrm{mM}$, c) Hyperkalemic stage with $\left[\mathrm{K}^{+}\right]_{\mathrm{o}}=10.0 \mathrm{mM}$.

(a)

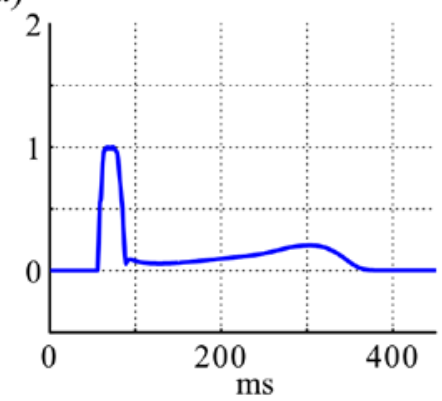

(b)

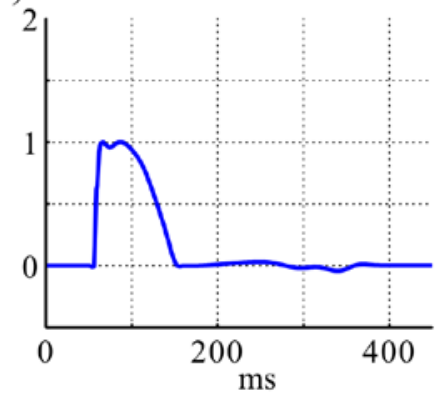

(c)

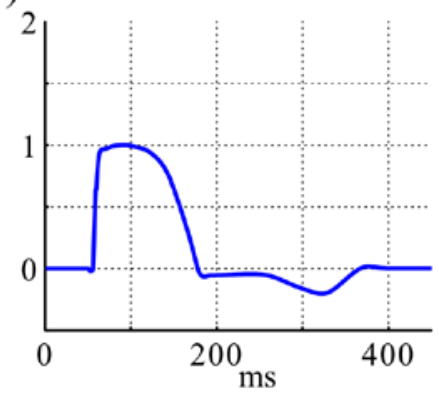

Figure 5. Pseudo-ECGs of simulated data with protocol 2 a) a) Basal conditions with $\left[\mathrm{K}^{+}\right]_{\mathrm{o}}=5.4 \mathrm{mM}$, b) Intermediate with $\left.\left[\mathrm{K}^{+}\right]_{0}=7 \mathrm{mM}, \mathrm{c}\right)$ Hyperkalemic stage with $\left[\mathrm{K}^{+}\right]_{\mathrm{o}}=10.0 \mathrm{mM}$.

$\mathrm{mV}$ of the basal condition to $-71.08 \mathrm{mV}$ with $\left[\mathrm{K}^{+}\right]_{\mathrm{o}}=10.0$ $\mathrm{mM}$. The $\mathrm{dV} / \mathrm{dt}$ and action potential duration (APD) were progressively reducing during the propagation of stimulus in hyperkalemic conditions, with a $10 \%$ of decrease of slope and $15 \%$ of APD with $\left[\mathrm{K}^{+}\right]_{0}=10.0 \mathrm{mM}$. In basal conditions, these parameters had not suffered changes. The changes of action potentials of wedge model are shown in Figure 4.

The reduction of CV prolonged activation sequence and, therefore QRS wave at equivalent proportion. Moreover, T-wave was decreasing progressively and started to be negative from $\left[\mathrm{K}^{+}\right]_{\mathrm{o}}=7 \mathrm{mM}$, as Figura 5 shows. This behaviour of $\mathrm{T}$ wave disappears when the protocol 1 is applied. In figure 6, pseudo-ECG obtained shows a high narrow $\mathrm{T}$ wave and its duration is lesser than in basal conditions.

\section{Discussion}

In the present study, simulations of hyperkalemic stages in combination with different patterns of stimulation have been proposed as mechanism of how patients with the same metabolic change show differences between their ECGs and symptoms. Delays of activation pattern, magnified by risk factors, are able to promote the necessary alterations to reproduce behaviour of Brugada syndrome. 


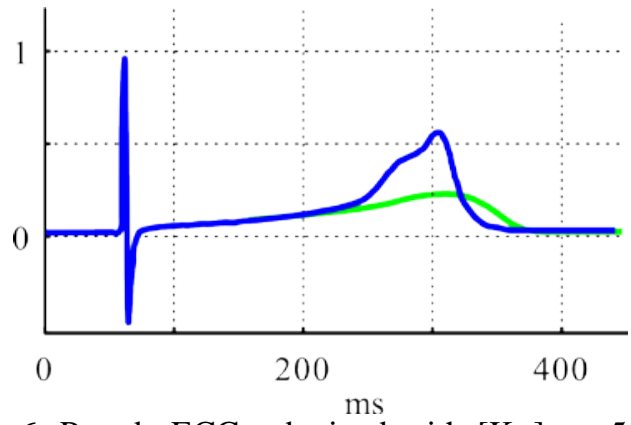

Figure 6. Pseudo-ECGs obtained with $[\mathrm{K}+] \mathrm{o}=5.4 \mathrm{mM}$ (green) and $[\mathrm{K}+]_{\mathrm{o}}=10.0 \mathrm{mM}$ (blue) in protocol 1 .

In our case, Hyperkalemia increased the resting potential and reduced the slope of $\mathrm{dV} / \mathrm{dt}$, leading to lower availability of sodium channels. This decreased t channels could be comparable to the genetic modifications of sodium channels that genuine patients with Brugada syndrome express $[1,6]$. Introducing a region of tissue with delay of activation, the duration of QRS wave was increased and T-wave became negative; typical features of Brugada syndrome. Also, this mechanism could be the responsible of other phenocopies of Brugada which have a low availability of sodium channels as hypothermia or acidosis in ischemic regions. Recently, data in patients with Brugada also it suggests that the depolarization theory is detectable in a large database of patients [12].

To achieve similar pseudo-ECGs to clinical electrograms, a wedge with transmural cell gradient was created. This model can represent a limitation in this study, to get more realistic ECGs, a whole organ model with detailed transmural gradients will be necessary. Such changes will be integrated into the model in future studies.

\section{Conclusion}

Delay of activation and heterogeneity of $\mathrm{CV}$ are the main reason of Brugada Phenocopy in a hyperkalemic

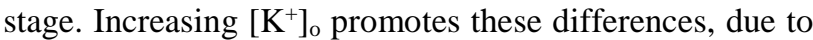
elevation of resting potential and lower availability of sodium channels.

\section{Acknowledgements}

Supported in part by grants from the Instituto de Salud Carlos III and Ministry of Economy and Competitiveness of Spain: (Grants PI13-01882, PI13-00903 and PI14/00857, Red RIC, PLE2009-0152 and Red de Terapia Celular); Spanish Society of Cardiology (Grant for Clinical Research in Cardiology 2015); Spanish Ministry of Economy and Competitiveness, Spain (Grant: IJCI-2014-22178, TEC2013-46067-R and TEC201350391-EXP).

\section{References}

[1] Antzelevich C, Brugada P, \& Borggrefe M. Brugada Syndrome. Report of the Second Consensus Conference. ACC Current Journal Review, 2005, vol. 14, no 6, p. 31.

[2] Riera ARP, Uchida AH, Schapachnik E, et al. Propofol infusion syndrome and Brugada syndrome electrocardiographic phenocopy. Cardiol J 2010;17:130135.

[3] Baranchuk A, Nguyen T, Ryu M, et al. Brugada phenocopy: new terminology and proposed classification. Annals of Noninvasive Electrocardiology, 2012;17:299314.

[4] Kes P. Hyperkalemia: a potentially lethal clinical condition. Acta Clinica Croatica, 2001, vol. 40, no 3, p. 215-225.

[5] Littmann L, Monroe MH, Taylor L, \& Brearley WD. The hyperkalemic Brugada sign. Journal of electrocardiology, 2007, vol. 40, no 1, p. 53-59.

[6] Wilde AA, Postema PG, DiDiego JM, et al. The pathophysiological mechanism underlying Brugada syndrome depolarization versus repolarization. J Mol Cell Cardiol 2010;49:543-553.

[7] Meregalli PG, Wilde AA, \& Tan HL. Pathophysiological mechanisms of Brugada syndrome: Depolarization disorder, repolarization disorder, or more?. Cardiovascular research, 2005, vol. 67, no 3, p. 367-378.

[8] O'Hara T, Virág L, Varró A, \& Rudy Y. Simulation of the undiseased human cardiac ventricular action potential: model formulation and experimental validation. PLoS Comput Biol, 2011, vol. 7, no 5, p. e1002061.

[9] Bishop MJ, Plank G, Burton RA, Schneider JE, Gavaghan DJ, Grau V, \& Kohl P. Development of an anatomically detailed MRI-derived rabbit ventricular model and assessment of its impact on simulations of electrophysiological function. American Journal of Physiology-Heart and Circulatory Physiology, 2010, vol. 298, no 2, p. H699-H718.

[10] Garcia-Molla V, Liberos A, Vidal A, Guillem MS, Millet J, Gonzalez A, Martinez-Zaldivar FJ, Climent AM: Adaptive step ODE algorithms for the 3D simulation of electric heart activity with graphics processing units. Comput Biol Med 2014;44:15-26.

[11] Courtemanche M, Ramirez RJ, Nattel S. Ionic mechanisms underlying human atrial action potential properties: Insights from a mathematical model. Am J Physiol Heart Circul Physiol 1998;275:H301-H321.

[12] Guillem MS, Climent AM, Millet J, Berne P, Ramos R, Brugada J, Brugada R. Spatiotemporal Characteristics of QRS Complexes Enable the Diagnosis of Brugada Syndrome Regardless of the Appearance of a Type 1 ECG. J Cardiovasc Electrophysiol. 2016 May;27(5):563-70

Address for correspondence.

Ismael Hernández Romero

Laboratorio de órganos y matrices bioartificiales Instituto de Investigación Sanitaria Gregorio Marañón Hospital GU Gregorio Marañón. Edificio Materno Infantil.

c〉 O'Donnell 48,

28009, Madrid, Spain

ismael.hdezromero@gmail.com 